\title{
Geringeres Honorarvolumen trotz höherer Fallzahl
}

Frau Dr. med. P. T., KV Bayern: Ich habe für das vierte Quartal 2011 ein geringeres Honorarvolumen (HV) erhalten als vor einem Jahr, obgleich sich die Fallzahl erhöht hat. Wie kann das sein?

Antwort: Das Honorarvolumen setzt sich zusammen aus dem Regelleistungsvolumen (RLV) multipliziert mit der Fallzahl (FZ) des Vorjahresquartals. Hinzu kommt die Summe der qualitätsgebundenen Zusatzvolumen (QZV). Diese setzt sich zusammen aus der Anzahl der verschiedenen QZV multipliziert mit der Fallzahl der Fälle, in denen das jeweilige QZV erbracht wurde. Der Berechnung zugrunde liegt die Abrechnung des Vorjahresquartals.

Als erstes muss die Richtigkeit der Fallzahl festgestellt werden. Ist diese nachvollzieh- bar, dann kann noch die im Bescheid errechnete Summe des RLV nachgerechnet werden.

Der nächste Schritt sind die QZV. Die QZV für Ultraschall oder psychosomatische Grundversorgung GOP 35100 und 35110 bedürfen der Genehmigung durch die KV. Hier gilt es, sofort zu prüfen, ob alle in Frage kommenden QZV genutzt werden.

In Bayern ergibt sich die derzeitige Summe der QZV aus der Anzahl der Fälle im 4. Quartal 2010, in denen ein QZV erbracht wurde. Dabei ist nicht die Häufigkeit der Erbringung der einzelnen Leistung, des einzelnen QZV von Bedeutung, sondern die Häufigkeit der Fälle, in denen ein einzelnes QZV erbracht wurde. Also: Zwei Fälle mit COPD oder Asth$\mathrm{ma}$, in denen das QZV Spirometrie erbracht wurde, rechnen für das laufende Quartal mit $2 \times$ QZV-Spirometrie. Hingegen $2 \times$ Spirometrie beim gleichen Behandlungsfall ergibt nur einmal ein QZV. Der Arzt erhält pro Fall für die in Vorquartalen abgerechneten QZV eine feste Punktzahl. Diese Punkte stehen zur Verfügung unabhängig von der Häufigkeit der Erbringung im Vorjahr.

Wenn also in einer Praxis in Bayern im 4. Quartal 2010 weniger QZV in weniger einzelnen Fällen erbracht wurde als im damaligen Bezugsquartal 4/09 ergibt sich automatisch ein Minus. Um dies zu vermeiden, gibt es nur eins: Es muss in jedem Quartal vom ersten Tag an aus wirtschaftlichen Gründen die Indikationsstellung für die zur Verfügung stehenden QZV Fall für Fall im Auge behalten werden.

\section{Krankenkasse will Stellungnahme zu meiner Abrechnung Wie soll ich mich verhalten?}

\section{Dr. med. F.-J.Z., Allgemeinarzt:}

Eine Krankenkasse hat mich zu einer Stellungnahme bezüglich einer Abrechnung von Leistungen aufgefordert. Was genau dahinter steht, wird nicht formuliert. Wie soll ich mich verhalten?

Antwort: Grundsätzlich hat eine Krankenkasse einem Vertragsarzt gegenüber keine Auskunftsberechtigung bezüglich seiner Abrechnung. Der Grund ist einfach: Zwischen Vertragsarzt und Krankenkassen besteht kein direktes Vertragsverhältnis.
Die Beziehungen bestehen zwischen Kassenärztlicher Vereinigung (KV) und Krankenkassen (KK) und zwischen KV und Vertragsarzt. Wenn also die KK auf Grundlage der Abrechnungsdaten, die sie von der KV erhalten hat, eine Stellungnahme vom Vertragsarzt möchte, dann muss die KK diese bei der KV anfordern unter Darlegung des Hintergrundes. Gehen Krankenkassen bei der Auswertung der ihnen zur Verfügung gestellten Daten beispielsweise von einer Falschabrechnung aus, müssen sie ent- sprechende Hinweise ausschließlich an die zuständige KV geben und gegebenenfalls Prüfanträge stellen. Bei direkten Anfragen der KK an den Arzt, sollte dieser sofort seine zuständige KV informieren und sich beraten lassen. Bei Betrugsvorwurf empfiehlt es sich unter Umständen sogar, einen versierten Anwalt seines Vertrauens dazwischen zu schalten. Ob berechtigt oder nicht: Schon der Verdacht auf einen Betrugsvorwurf ist so schwerwiegend, dass es gilt, auch den geringsten juristischen Fehler zu vermeiden! 